\title{
Nonthermal Transport of Energy Driven by Photoexcited Carriers in Switchable Solid States of GeTe
}

\author{
R. Gu, ${ }^{1}$ T. Perraulte, ${ }^{1}$ V. Juvé, ${ }^{1}$ G. Vaudel, ${ }^{1}$ M. Weis $\odot,{ }^{1}$ A. Bulou, ${ }^{1}$ N. Chigarev, ${ }^{2}$ A. Levchuk $\odot,{ }^{1}$ \\ S. Raetz $\odot,{ }^{2}$ V.E. Gusev $\odot,{ }^{2}$ Z. Cheng, ${ }^{3}$ H. Bhaskaran, ${ }^{3, *}$ and P. Ruello ${ }^{1, \dagger}$ \\ ${ }^{1}$ Institut des Molécules et Matériaux du Mans, UMR 6283 CNRS, Le Mans Université, Le Mans 72085, France \\ ${ }^{2}$ Laboratoire d'Acoustique de l'Université du Mans (LAUM), UMR 6613, Institut d'Acoustique - Graduate School \\ (IA-GS), CNRS, Le Mans Université, Le Mans, France \\ ${ }^{3}$ Department of Materials, University of Oxford, United Kingdom
}

(Received 17 September 2020; revised 26 May 2021; accepted 17 June 2021; published 22 July 2021)

\begin{abstract}
Phase-change alloys have seen widespread use, from rewritable optical disks to current interest in their use in emerging neuromorphic computing architectures. In spite of this enormous commercial interest, the physics of the carriers in these materials is still not fully understood. Here, we describe the time and space dependence of the coupling between photoexcited carriers and the lattice in both the amorphous and crystalline states of one phase-change material, GeTe. We study this material using a time-resolved optical technique called the picosecond acoustic method to investigate the in situ thermally assisted amorphous-tocrystalline phase transformation in GeTe. Our work reveals a clear evolution of electron-phonon coupling during the phase transformation, as the spectra of photoexcited acoustic phonons in the amorphous ( $a$ $\mathrm{GeTe})$ and crystalline ( $\alpha$-GeTe) phases are different. In particular, and surprisingly, our analysis of the photoinduced acoustic pulse duration in crystalline GeTe suggests that part of the energy deposited during the photoexcitation process takes place over a distance that clearly exceeds that defined by the skin depth of the pump light. Alternatively, the photoexcitation process remains localized within that skin depth in the amorphous state. We then demonstrate that this is due to supersonic diffusion of photoexcited electronhole plasma in the crystalline state. Consequently, these findings prove the existence of the nonthermal transport of energy, which is much faster than lattice heat diffusion.
\end{abstract}

DOI: 10.1103/PhysRevApplied.16.014055

\section{INTRODUCTION}

Phase-change materials (PCMs) have become a critical aspect of advanced computing architectures [1], ranging from arithmetic operations [2-4] to neuromorphic computing [5-7] and, more recently, matrix-vector multiplications for machine-learning and artificial-intelligence applications [8-10]. In spite of their large deployment in industry and products, and a wealth of studies [11-13], the physics of the dynamics of phase transformation is still under intense study [14]. These technologies are underpinned by a reversible phase transformation from an amorphousto-crystalline phase that is thermally, optically, or electrically assisted [11-14]. The need for fast writing-erasing processes in emerging $\mathrm{GHz}-\mathrm{THz}$ information technologies will benefit from ultrafast photoinduced transformation. However, this is presently not possible, and mastering this technology requires a better understanding of electron and phonon dynamics at short time- and space

*harish.bhaskaran@materials.ox.ac.uk

†pascal.ruello@univ-lemans.fr scales in nonequilibrium states, e.g., after a photoexcitation process [12-16]. While phase transformation was initially described as a thermally assisted process, recent debate about the crucial role of photoexcited carriers in the amorphization process ( $\alpha$-GeTe to $a$-GeTe) has raised the question of a potentially complex nonthermal process $[12,16,17]$, which calls for clarification. In this photoinduced transformation process, the photoexcited carriers are suspected to modify the interatomic potential in a nonthermal way, leading, under some circumstances, to lattice instability $[12,16,17]$. Photoexcited carrier-lattice coupling is at the core of the crucial mechanism for the transformation of light energy into lattice energy and represents the necessary step for phase transformation. Contradictory assumptions of the characteristic spatial extension of coupling between photoexcited carriers and phonons currently exist. It is considered that the photoexcited carrier-lattice coupling in PCMs is restricted to the optical skin depth (light penetration) [12,16-20], while another report suggests a possible ballistic electron effect [21], without any clear and direct conclusion related to this crucial physical phenomenon. Using a time-resolved optical method 
[22-24], we demonstrate that photocarriers supersonically diffuse in the crystalline phase $\alpha$-GeTe over a distance that is around 7 times the optical skin depth, i.e., the photoexcited carriers transport energy via a nonthermal process around 7 times deeper than the depth of light absorption. However, we show that this supersonic electron-hole plasma expansion is not effective in the amorphous state $a$-GeTe. This technique, called the picosecond acoustic method, enables depth profiling of the coupling between photoexcited carriers and the lattice. Such a method has already been used to show how photoexcited carriers rapidly diffuse nonthermally out of the optical skin in GaAs $[25,26]$ or Ge [27] semiconductors. It has also been employed to reveal ultrafast nonlocal heating in metals [28-30]. Time-resolved optical techniques, such as coherent optical-phonon spectroscopy, have already been used to probe the amorphous-to-crystalline phase transformation, but this method is only sensitive to optical phonons (i.e., unit cell) and cannot map photoexcited carrier diffusion [31,32]. Photoinduced strain studies have already been reported in $\mathrm{Ge}_{2} \mathrm{Sb}_{2} \mathrm{Te}_{5}$ compounds [17,18,33]. Some differences in the acousto-optic signals have been reported $[18,33]$, but this nonthermal supersonic electronic diffusion contribution to acoustic pulse broadening was not discussed or observed. The crucial parameter in this study, besides the high sensitivity of optical measurements, is the choice of the PCM thin-film thickness. Most previous studies [16-21] concern thin films, the thicknesses of which are typically of the same order as the optical-pump lightpenetration depth $(\sim 30 \mathrm{~nm})$ in the crystalline PCM. These thin thicknesses are usually chosen for necessary experimental constraints associated with time-resolved x-ray or electron-diffraction [16,17,19-21]. For such thin layers, there is a natural in-depth confinement of the photoexcited electron-hole plasma, preventing the electron-hole plasma from expanding. Here, we perform in situ pumpprobe experiments on a much thicker film, with a GeTe film having a thickness of $380 \mathrm{~nm}$, to let the electron-hole plasma expand over a distance of around $200-400 \mathrm{~nm}$, as we will show. We then provide evidence of ultrafast nonthermal transport of energy that is much faster than the ballistic acoustic phonon propagation, which sets a limit for phonon thermal transport.

\section{EXPERIMENTAL METHODS}

The principle of the pump-probe experiments described here is shown in Fig. 1(a), where the light-matter interaction leads to the generation of the longitudinal acoustic phonon (LA), the spectrum (pulse duration) of which provides information about the photoexcited carrier dynamics, in particular, about the hot-carrier diffusion. A description of the nanostructure under investigation is shown in Fig. 1(b). The GeTe sample is grown through a physical deposition method ( $\mathrm{rf}$ sputtering). The sample has a thickness of around $380 \mathrm{~nm}( \pm 10 \mathrm{~nm})$. It is covered by a protecting thin $(10 \mathrm{~nm})$ transparent ITO layer. The stoichiometry of GeTe is 50:50 and it is deposited on $\mathrm{Si}$ substrate with a $\mathrm{SiO}_{2}$ buffer layer. The pump-probe method is based on a Ti:sapphire femtosecond laser that delivers $\mathrm{nJ}$ pulses of around $200 \mathrm{fs}$ in duration. The repetition rate is $80 \mathrm{MHz}$. The experiments are conducted with a two-color pump-probe scheme with pump laser radiation centered at $830 \mathrm{~nm}(1.5 \mathrm{eV})$ or at its second harmonic, $415 \mathrm{~nm}(3$ $\mathrm{eV}$ ), obtained by optical second-harmonic generation in a BBO crystal (Beta Barium Borate). The probe-beam wavelength is always fixed at $583 \mathrm{~nm}(2.12 \mathrm{eV})$. The focusing diameter of the pump and probe beams is about 14 and $8 \mu \mathrm{m}$. The pump and probe are focused on the GeTe surface with a quasinormal incidence. $830 \mathrm{~nm}$ is the fundamental wavelength of a Ti:sapphire oscillator, while the probe-beam wavelength is controlled thanks to an optical parametric oscillator. For picosecond acoustic experiments versus temperature, a Linkam furnace is used to realize in situ temperature-dependence measurements.

In this experiment, light energy is absorbed and distributed over a characteristic distance, which leads to the generation of a strain pulse. The latter travels back and forth (after reflection on the substrate) through the layer and periodic acoustic echoes are detected at times $t_{1}$ and $t_{2}$, as indicated in Fig. 1(c). Our goal is to probe how energy absorbed by the electronic subsystem after femtosecond excitation is transferred to the phonon subsystem via electron-phonon coupling in the amorphous and crystalline states. Investigations are then conducted within the perturbative approach, i.e., we aim to investigate the change in physical properties under a thermally assisted phase transformation and not under light-induced phase transformation. As a consequence, careful measurements are done by selecting the proper pump and probe fluences to prevent any transformation of GeTe under light excitation. It is known that a photoassisted excitation process can transform the amorphous phase into the crystalline phase and vice versa [16,34]. To ensure that we do not induce the amorphous-to-crystalline phase transformation (and the reverse one) under the action of the pump beam, we calibrate our measurements, as described in Note $\mathrm{S} 1$ within the Supplemental Material [35]. We show that working with pump fluences ranging from 2.5 to $5 \mathrm{~mW}$, i.e., $60 \mu \mathrm{J} / \mathrm{cm}^{-2}$ (for our laser repetition rate of $80 \mathrm{MHz}$ ), allows us to remain below the optical threshold (see Figs. 1 and 2 in Note S1 within the Supplemental Material [35]).

\section{RESULTS}

A typical transient-optical-reflectivity signal is shown in Fig. 1(c) for the case of amorphous GeTe. We identify first a sharp variation just after pump excitation (time $t_{0}$ ), corresponding to excitation of the electronic subsystem followed by rapid electron-hole-phonon thermalization. 


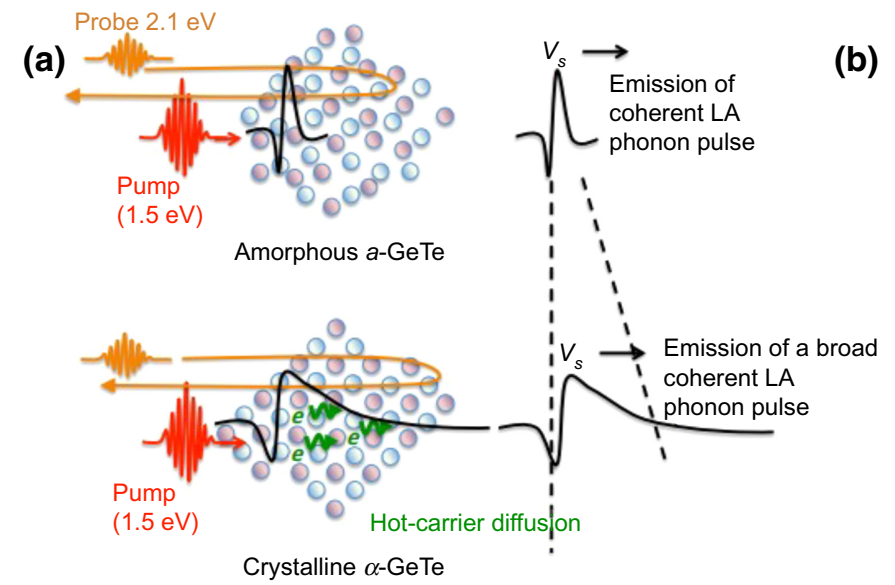

(b)
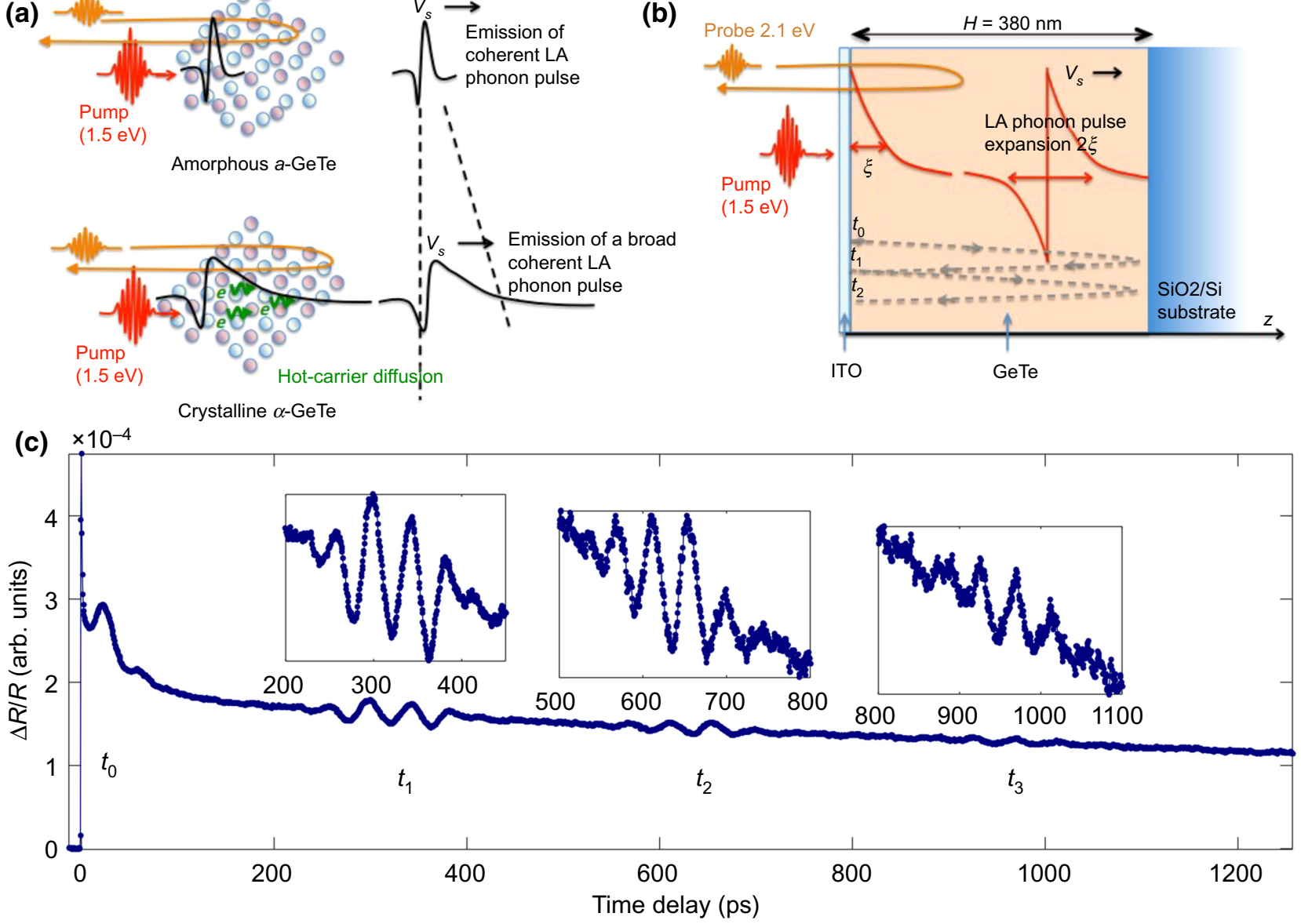

FIG. 1. (a) Sketch of photoexcitation of GeTe in the amorphous and crystalline phases, where the photoinduced acoustic phonon pulses have strong differences related to the electronic properties of the material. (b) Description of the 380-nm-thick thin GeTe film studied with time-resolved optical measurements. Femtosecond-laser pump pulse generates a strain pulse near the surface that travels back and forth in the film, as depicted by arrows. These acoustic pulses are monitored in the time domain thanks to a delayed probe pulse. (c) Typical transient-optical-reflectivity signals obtained with pump radiation at $415 \mathrm{~nm}$ and probed at $583 \mathrm{~nm}$ in the amorphous phase, revealing clearly three acoustic also shown in insets.

Slight modulation of the transient-optical-reflectivity signal is observed over the time range $(\sim 0-50 \mathrm{ps})$, with more clearly pronounced oscillations at around 300,600 , and $900 \mathrm{ps}$. The periodic nature of these features $\left(t_{2} \approx 2 t_{1}\right.$ and $t_{3} \approx 3 t_{1}$ ) typically indicates that they correspond to acoustic echoes which come from an acoustic pulse that travels back and forth in the thin film, consistent with our previous description of the phenomenon in Fig. 1(b). As seen in the following (Fig. 2), a drastic change to the transient reflectivity signal is revealed when we thermally induce the amorphous-to-crystalline phase transition. We start with the amorphous GeTe film, and the temperature is increased to $300{ }^{\circ} \mathrm{C}$ at a rate of $5 \mathrm{~K} / \mathrm{s}$ to achieve the amorphousto-crystalline transformation. An optical contrast change is observed, as expected, after the transformation and shown in Fig. 2(a). The thermally induced amorphous-tocrystalline phase transformation is verified in parallel using Raman spectroscopy. Raman spectra are recorded with a
T64000 Jobin-Yvon spectrometer under a microscope with an objective of $50 \times$. The laser wavelength is $647.1 \mathrm{~nm}$. The incident power on the sample is $0.2 \mathrm{~mW}$. The results are shown in Fig. 2(b). The characteristic bands of the amorphous phase are indicated by letters ii, iii and iv, and are consistent with the literature [36,37]. When GeTe is heated to $300{ }^{\circ} \mathrm{C}$ (above the crystallization temperature), the characteristic band of the crystalline phase [band i] is revealed and is consistent with prior reports in the literature [36,37]. Notably, heating the sample at $400{ }^{\circ} \mathrm{C}$ makes bands iii and iv reappear, which could correspond to partial melting followed by the amorphous phase formation after cooling down the sample. The time-resolved signals are shown in Figs. 2(c) and 2(d). Up to $150^{\circ} \mathrm{C}$, the transientoptical-reflectivity signal does not evolve significantly, as witnessed by the nearly unchanged shape of the acoustic echo [Fig. 2(c)]. Above this temperature, the signal drastically evolves up to the studied maximum of $300^{\circ} \mathrm{C}$. We 

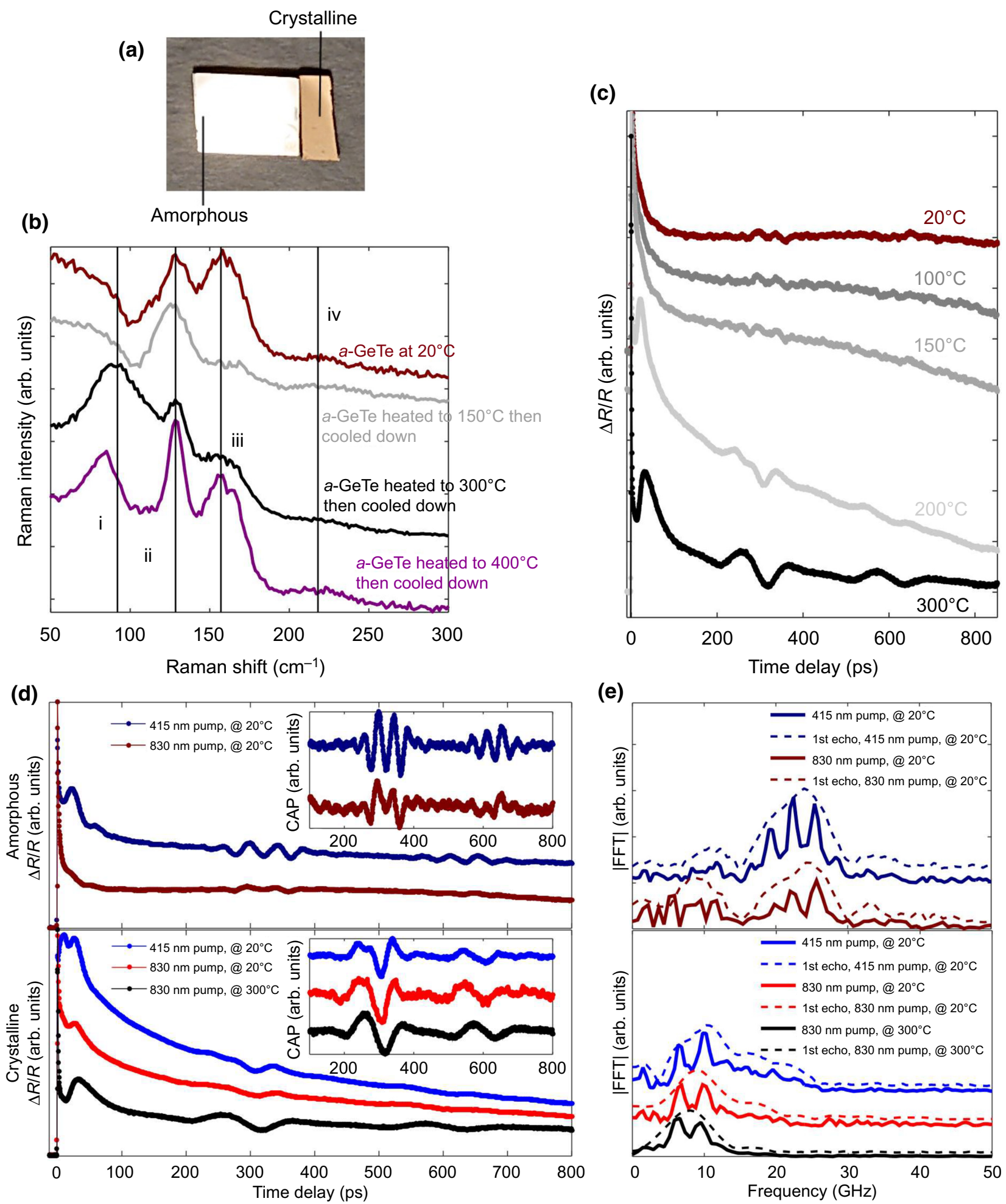

FIG. 2. (a) Image with a white-light source showing optical contrast between amorphous and crystalline GeTe. (b) Raman spectra recorded as a function of temperature. (c) Transient-optical-reflectivity signals versus temperature during the amorphous-to-crystalline phase transformation (pump@830 nm). (d) Comparison of transient-optical-reflectivity signals obtained with two pump photon energies, $830 \mathrm{~nm}(1.5 \mathrm{eV})$ and $415 \mathrm{~nm}(3 \mathrm{eV})$, for both amorphous (at room temperature only) and crystalline phases (at room temperature and at $300{ }^{\circ} \mathrm{C}$ ). Inset shows acoustic echoes only. (e) Fast Fourier transform (FFT) of acoustic signals shown in inset of (d). FFT of the first acoustic echo (envelope of the signal depicted with dashed lines) compared with FFT of the full signal (solid lines). 
observe a significant change in the shape of the acoustic phonon signal with temperature, which is also a clear signature of a phase transformation [Figs. 2(c) and 2(d)], as already shown for various structural phase transitions $[38,39]$. With this measurement, we also find evidence of a clear evolution of the photoexcited carrier-relaxation time [Figs. 3(a) and 3(b) within the Supplemental Material [35] ], as usually observed during a phase transformation $[40,41]$ and discussed in Note S2 within the Supplemental Material [35]. Finally, once crystallization is thermally achieved above $300^{\circ} \mathrm{C}$, we cool the sample and repeat the time-resolved experiments over the range $20-300^{\circ} \mathrm{C}$ to confirm the stability of the crystalline phase [see Fig. 3(c) within the Supplemental Material [35] ]. Experiments are also conducted at room temperature with both states of GeTe with a pump energy of $3 \mathrm{eV}$. The transient-opticalreflectivity signals obtained for both pump energies are shown in Fig. 2(d). For comparison, we also show the signal obtained at $300^{\circ} \mathrm{C}$ for $\alpha$-GeTe. Coherent acoustic phonons are shown in the inset of Fig. 2(d) once the baseline is removed, and the spectrum of acoustic phonons obtained with a fast Fourier transform (FFT) is presented in Fig. 2(e). The signals obtained with a pump photon of $3 \mathrm{eV}$ confirm the drastic evolution of the acoustic pulse shape when the material becomes crystalline. Less pronounced is the pump photon-energy dependence of the acoustic pulse shape either in the amorphous or in the crystalline state. The striking point we notice about the Fourier components of coherent acoustic phonon signals [Fig. 2(e)] is the redshift of the main component of the spectrum in the case of the crystalline state comparatively to the amorphous state: while centered at around $25 \mathrm{GHz}$ for the amorphous state, it redshifts down to around $10 \mathrm{GHz}$ for the crystalline state. The spectrum for the signal obtained at $300^{\circ} \mathrm{C}$ is similar to that obtained at room temperature, with only a slight attenuation of the highest-frequency components, but the acoustic pulse duration remains unchanged. The sequence of periodic peaks appearing in the spectrum of the full signal is because the FFT is realized with successive echoes, so that the spectrum is the result of a convolution of the spectrum of a single acoustic echo [envelope of the spectrum shown as dashed lines in Fig. 2(e)], with the spectrum of a periodic signal associated with the back and forth travel of acoustic phonons. The repetition in time of these acoustic signals gives rise in the frequency domain to a sequence of frequencies, $f_{n}$, with $f_{n}=n V / 2 H(n=1,2, \ldots)$, where $V$ is the velocity of sound and $H$ is the thickness of GeTe. This sequence allows us to estimate, with good precision, the velocity of sound in the amorphous $\left(V_{a} \approx 2400 \mathrm{~m} / \mathrm{s}\right)$ and in the crystalline $\left(V_{\alpha} \approx 2500 \mathrm{~m} / \mathrm{s}\right)$ state (see Note S3 and Fig. 4 within the Supplemental Material [35]). Considering the bulk-modulus values for $\alpha$-GeTe either calculated $(T=0 \mathrm{~K})$, with $B \approx 46 \mathrm{GPa}$ [42] and $B \approx 44.3$ $\mathrm{GPa}$ [43], or measured ( $T=300 \mathrm{~K}), B \sim 49.9 \mathrm{GPa}$ [44], the longitudinal velocity of sound becomes included in the range $V \approx \sqrt{B / \rho} \approx 2680-2850 \mathrm{~m} \mathrm{~s}^{-1}$ (mass density $\rho=6140 \mathrm{~kg} \mathrm{~m}^{-3}$ ), which is in reasonable agreement with our measurements. However, it is worth mentioning that a much smaller value of $1900 \mathrm{~m} \mathrm{~s}^{-1}$ is measured by nuclear inelastic scattering methods for $\alpha$-GeTe [45]. We believe that the large discrepancies might come from different microstructures of the samples.

In the following, we discuss the acoustic echo duration and shape to demonstrate that the signals can be understood only if we consider nonthermal transport of energy achieved by hot carriers in the crystalline phase, due to supersonic electron-hole plasma expansion.

\section{DISCUSSION}

In a semi-infinite solid, when light impinges on the free surface and leads to photoinduced stress with the in-depth profile defined by pump-light penetration (i.e., by skin depth $\xi_{\text {pump }}$ ), the existence of the mechanically free surface leads to emission of an acoustic strain pulse, $\eta(z, t)$, with a bipolar shape and $\eta(z, t)=-\eta_{0} \operatorname{sgn}(z-$ $\left.V_{S} t\right) \exp \left(-\left|z-V_{S} t\right| / \xi\right)$ [this bipolar pulse is shown in Fig. 1(b)]. As discussed in Fig. 1(b), this strain pulse $\eta(z, t)$ travels back and forth through the thin film. This strain field is not directly detected, since in the time domain there is a temporal convolution of this strain pulse, with the indepth distribution of the electric field of the probe beam represented by $e^{2 i k_{0} \tilde{n}_{\mathrm{GeTe}} z}$ in following Eq. $1[22,23,25]$. The theoretical transient-optical reflectivity is $\Delta R / R=$ $2 \operatorname{Re}(\delta r / r)$ with $[22,23,25]$

$$
\begin{aligned}
\delta r / r \simeq & \rho+i \delta \phi \simeq-2 i k_{0} \delta z+\frac{4 i k_{0} \tilde{n}_{\mathrm{GeTe}}}{\left(1-\tilde{n}_{\mathrm{GeTe}}^{2}\right)} \frac{d \tilde{n}_{\mathrm{GeTe}}}{d \eta} \\
& \times \int_{0}^{\infty} \eta(z, t) e^{2 i k_{0} \tilde{\mathrm{GeTe}} z} d z
\end{aligned}
$$

where $k_{0}=2 \pi / \lambda$ is the probe wave vector in air, $\eta(z, t)$ is the coherent acoustic-phonon-strain field propagating perpendicularly to the surface of the sample, $\tilde{n}_{\mathrm{GeTe}}$ is the complex refractive index of GeTe [13,46], and $d \tilde{n}_{\mathrm{GeTe}} / d \eta$ is the photoelastic coefficient. In this model, we neglect the very thin transparent layer of ITO, so $z=0$ corresponds to the air-GeTe interface. Considering the longitudinal velocity of sound of $6500 \mathrm{~m} \mathrm{~s}^{-1}$ in ITO [47], the back and forth traveling of the acoustic pulse inside this thin layer contributes only to a delay of about $3 \mathrm{ps}$, which is much shorter than the detected pulse duration of hundreds of picoseconds. In other words, the presence of the capping layer does not noticeably broaden the photogenerated acoustic pulses because the ITO layer thickness is much smaller than the characteristic in-depth distribution of the photoinduced strain field and the velocity of sound in the ITO layer is higher than that in GeTe. In Eq. (1), the first term corresponds to the contribution of the surface displacement $(\delta z)$ of the film at $z=0$. The second term of Eq. (1) is 
the photoelastic contribution (due to modification of the refractive index of the GeTe material induced by the strain field of the coherent acoustic phonons). The upper limit of integration is taken to be infinite, which is justified, since the optical-probe penetration is much less than the film thickness. The photoelastic coefficient is estimated according to $d \tilde{n}_{\mathrm{GeTe}} / d \eta=\left(d \tilde{n}_{\mathrm{GeTe}} / d E\right)(d E / d \eta)$, with $E$ as the energy at which the detection process is realized. $d \tilde{n}_{\mathrm{GeTe}} / d E$ is estimated on the basis of tabulated data (see Fig. 5 in Note S4 within the Supplemental Material [35]), and $d E / d \eta$ is the deformation-potential parameter. We currently do not know $d E / d \eta$, so, in this simulation, we can simulate the shape and acoustic pulse duration of the signal but not the absolute value for the magnitude of $\Delta R / R$. The calculation is shown in Fig. 3(a). It shows that the detected acoustic phonon signal in the amorphous phase is reasonably reproduced within that model. Our calculation does not perfectly reproduce the shape of the acoustic echo, probably because the photoelastic coefficients are slightly different from our estimation. Despite the discrepancies concerning the acoustic echo shape, we are able to see that the pulse duration in the amorphous state is reasonably reproduced. This indicates that the assumption of the photoinduced-strain in-depth distribution described

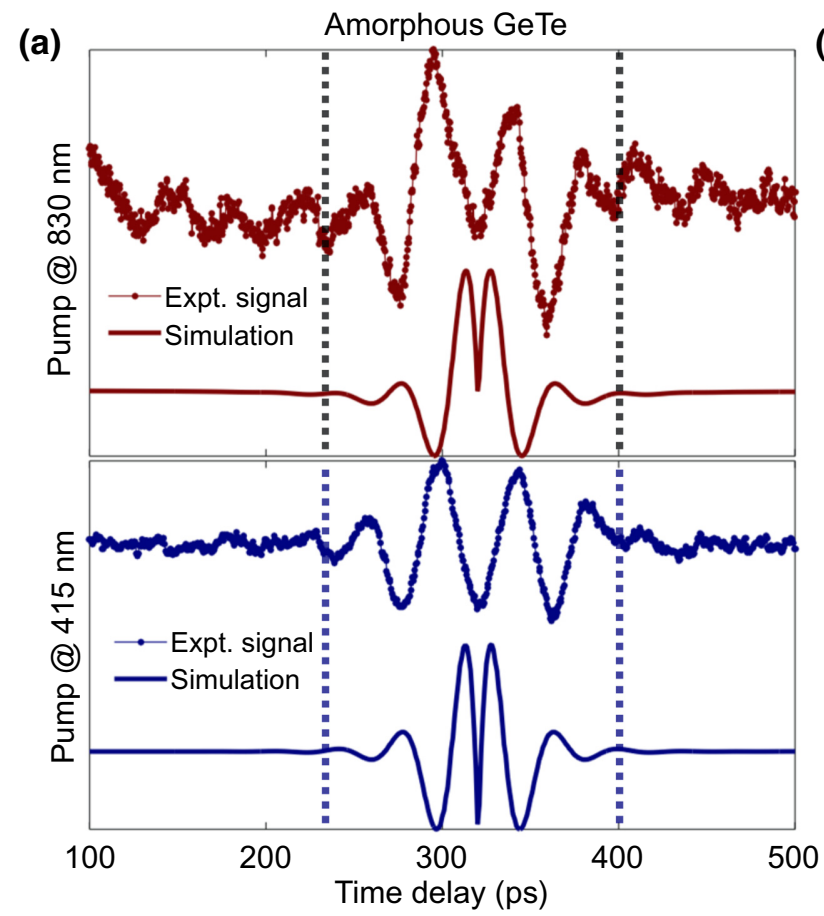

(b)
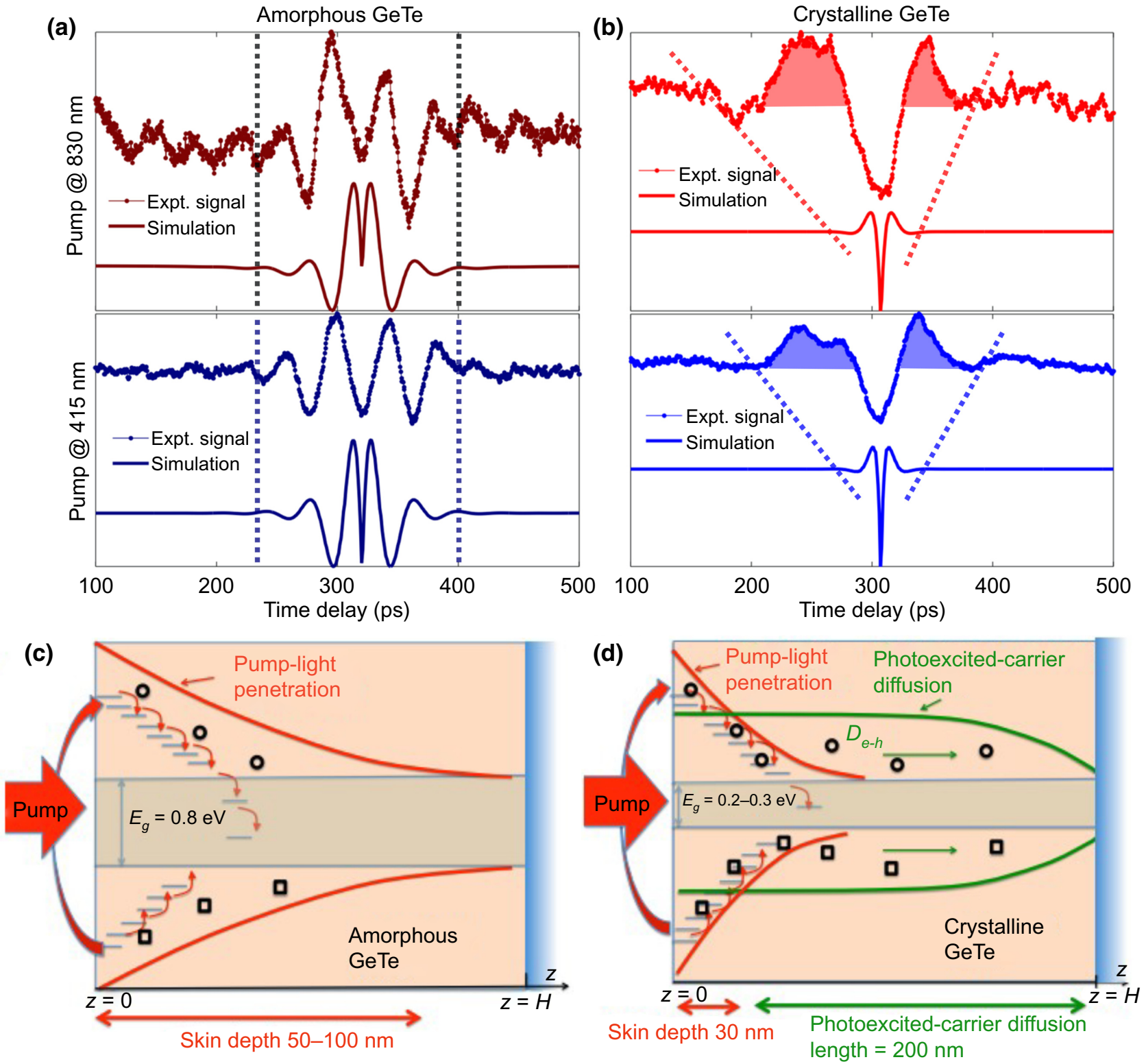

FIG. 3. Calculation of coherent acoustic phonon signals in amorphous (a) and crystalline (b) states, when the strain pulse is defined by the optical skin depth of pump radiation [see Eq. (1) in the main text]. (c) Sketch of the spatial distribution of photoexcited carriers in the $a$-GeTe and $\alpha$-GeTe phases. While in the amorphous state, photoexcited carriers couple to the lattice within the skin depth of the optical pump, these carriers diffuse at a much longer distance away from this skin depth in the crystalline state. Circles and squares are symbols for electrons and holes, respectively. 
by the optical-pump penetration is acceptable. In other words, this strongly suggests a local electron-hole-lattice coupling in the amorphous phase. We carry out the same calculation for the crystalline phase, using the same procedure and employing the optical properties provided by the same Refs. [13,46] [see Fig. 3(b)]. For this crystalline state, we immediately see a large discrepancy between calculation and experimental measurements, for both the 415- and 830-nm pump wavelengths. Due to reduction of the band gap from $E_{g} \sim 0.8 \mathrm{eV}$ (amorphous) to $E_{g} \sim$ $0.2-0.3 \mathrm{eV}$ (crystalline), the imaginary part of the refractive index significantly increases in the crystalline state. As a consequence, using Eq. (1), if the strain profile is solely defined by the in-depth penetration of the pump light, we predict a much shorter pulse duration than the experimental one. The fact that the experimental pulse duration is much longer than that calculated (i.e., shift of the phonon spectrum towards the low-frequency range) suggests that strain is induced over a distance exceeding that of pumplight penetration, i.e., it is nonlocal in nature. Considering the frequency of the emitted coherent acoustic phonon [ $<10 \mathrm{GHz}$, see Fig. 2(e)], this broadening effect cannot be associated with rapid heat diffusion out of the skin layer. If a light-induced heating effect is responsible, this would mean that the generation of strain would be driven by a thermal process, i.e., by a thermoelastic process [22-24]. We note that such thermoelastic processes, well known in metals [22,23], are based on a rapid heating of the lattice induced by light absorption. Such heating leads to lattice expansion and, consequently, to strain-pulse generation. It is important to know the spatial scale at which this process takes place. For that, it is necessary to evaluate the phonon heat flow in $\alpha$-GeTe and to see whether lattice heating takes place only within the optical skin depth of the pump laser or if phonon heat transport can occur over a distance larger than the pump skin depth, $\xi_{\text {pump }}$, and hence, extend the strain field over the skin depth. Within this thermoelastic process, typical times for acoustic phonons to leave the region where the pump light is absorbed are defined by $\tau \approx 2 \xi_{\text {pump }} / V_{S}$. During time $\tau$, incoherent phonons can diffuse over a typical distance of $L_{\mathrm{th}} \approx \sqrt{D_{\text {heat }} \tau}$, where $D_{\text {heat }}$ is the heat-diffusion coefficient. As a consequence, the ratio between the characteristic thermal diffusion length and the optical skin depth of pump radiation becomes $L_{\text {th }} / \xi_{\text {pump }} \approx \sqrt{2 D_{\text {heat }} /\left(\xi_{\text {pump }} V_{S}\right)}$. For $\alpha$-GeTe we have, at room temperature, $D_{\text {heat }}=2 \times 10^{-6} \mathrm{~m}^{2} \mathrm{~s}^{-1}[48,49]$, and taking $V_{S}$ as $\approx 2500 \mathrm{~m} / \mathrm{s}$ we obtain $L_{\text {th }} / \xi_{\text {pump }} \approx 0.2<1$. This means that heat does not have time to escape the skin-depth region characterized by $\xi_{\text {pump }}$ before longitudinal acoustic phonons leave the same region (we can say that heat diffusion is subsonic). Thus, incoherent phonon heat diffusion cannot explain our observed acoustic pulse broadening. A spatial broadening of the traveling acoustic pulse induced by acoustic phonon dispersion can also be ruled out. In the case where the acoustic pulse is emitted only at the skin depth, the characteristic width of the coherent acoustic-pulse-frequency spectrum would be given by $f \approx V / \xi \approx 100 \mathrm{GHz}$. Considering the dispersion curves found in Ref. [42], we see that, even at a highfrequency component $(100 \mathrm{GHz})$, the phase velocity is nearly the same as the one we can deduce at the lowest frequency (Brillouin zone center). Consequently, the dispersion effect, i.e., the difference in the propagation time between the highest- and lowest-frequency components, over the relevant propagation distance (less than $1 \mu \mathrm{m}$ ) can potentially broaden the coherent acoustic pulse only by a few picoseconds, which is much shorter than the duration of the detected acoustic pulse approaching $200 \mathrm{ps}$ [see inset of Fig. 2(d)]. Moreover, analysis of successive echoes shows that the acoustic pulse duration does not drastically change over time at the considered propagation distances [see Fig. 2(d)], demonstrating that attenuation also plays a minor role in controlling the acoustic pulse duration at such short propagation distances and relatively low frequencies. We also note that the acoustic pulse obtained for the signal measured at $300^{\circ} \mathrm{C}$ [see Fig. 2(d)] has a similar duration to that measured at room temperature, confirming that the contribution of temperature (i.e., phonon-phonon collision dynamics) to this broad coherent acoustic-phonon-pulse spectrum is not predominant and does not even exist. Finally, we can also show that nonlinear acoustic effects have a negligible effect on pulse broadening, since the distance of propagation of the photogenerated acoustic pulse in our experiment is shorter than the characteristic distance over which the nonlinear effects develop (see Note S5 within the Supplemental Material [35]).

Having excluded thermal, dispersion, attenuation, and nonlinear acoustic effects, it is important to remember that the transport of energy, in particular, for photoexcited materials, is not achieved only by incoherent phonons (heat). Some photoexcited carriers can contribute to this transport of energy. Such a phenomenon has already been observed in the semiconductors GaAs [25,26] and Ge [27] and is attributed to a supersonic expansion of electron-hole plasma. In this case, the photoexcited carriers rapidly diffuse over the conduction and valence bands and couple to the lattice over a distance longer than the pump skin depth. In the particular case of these semiconductors, GaAs and $\mathrm{Ge}$, the coherent acoustic phonons are generated through the electron-hole-acoustic-phonon deformation-potential coupling mechanism (nonthermal process). This mechanism is possible only if the photoexcited carriers do not recombine before they diffuse out of the skin depth. In this scenario, the characteristic acoustic phonon pulsation driven by this rapid plasma expansion is given by $\omega \approx$ $V_{S}^{2} / D_{e-h}$, where $D_{e-h}(h$, hole; $e$, electron) is the carrierdiffusion coefficient $[23,50]$. In our case, if we assume this process to be the driving one, this characteristic frequency is given by the inverse of the duration of the acoustic pulse, i.e., $\omega \approx 1 / \Delta t \approx 10 \mathrm{GHz}$ [this characteristic 
frequency corresponds to the maximum of the spectrum of a single acoustic echo, as shown in Fig. 2(e)]. Consequently, we deduce from our measurements a photoexcited carrier-diffusion coefficient of $D_{e-h} \approx 0.6 \times 10^{-3} \mathrm{~m}^{2} \mathrm{~s}^{-1}$. This value is characteristic for electron and hole diffusion in semiconductors [51]. To show that this value is actually relevant for crystalline GeTe, and if we assume the Einstein relation to be valid, then we can also evaluate the expected diffusion coefficient, $D_{e-h}$, in $\alpha$-GeTe from the electrical mobility tabulated in the literature $\left(\mu_{h, e} \approx 100-200 \mathrm{~cm}^{2} \mathrm{~V}^{-1} \mathrm{~s}^{-1}\right)$ [52]. It is worth mentioning that this mobility is 4 orders of magnitude larger than in the amorphous phase, where a typical value of $\mu_{h, e} \approx 0.1-0.2 \mathrm{~cm}^{2} \mathrm{~V}^{-1} \mathrm{~s}^{-1}$ is found [53]. Using such an approach, we arrive at a value for $D_{e-h} \approx \mu_{h, e} k_{B} T / q \approx$ $0.2-0.4 \times 10^{-3} \mathrm{~m}^{2} \mathrm{~s}^{-1}$ at $T=300 \mathrm{~K}\left(k_{B}\right.$ and $q$ are the Boltzmann constant and the elementary charge of a carrier, respectively). Interestingly, this value is consistent with that deduced from the analysis of the diffusive origin of acoustic echo broadening. Thus, our observation strongly supports that photoinduced stress is governed by rapid (supersonic) photoexcited carriers in crystalline $\alpha$-GeTe. A sketch is shown in Fig. 3(b), whereas the photoexcited electrons and holes are confined within the skin depth for the amorphous phase (and recombine nonradiatively to produce heat), the photoexcited carriers rapidly diffuse out of the skin depth with a characteristic diffusion coefficient, $D_{e-h}$, in the crystalline state. This supersonic diffusion takes place over a typical distance of $L_{D} \approx \sqrt{D_{e-h} \Delta t} \approx 200 \mathrm{~nm}$, if we take $D_{e-h} \approx 0.6 \times 10^{-3} \mathrm{~m}^{2} \mathrm{~s}^{-1}$. This means that photoexcited carriers can diffuse over a significant distance and are responsible for nonthermal transport energy in crystalline GeTe, as we sketch in Figs. 1(a) and 3(b). As a final element for discussion, we would like to focus our attention on the asymmetry of the detected pulse, as underlined by the dashed area in Fig. 3(b). As demonstrated in previous works (Chapters 2-4 in Refs. [23-27]), the initially rapid (supersonic) diffusion of photoexcited carriers from the free surface gives rise to an asymmetric strain pulse, as we sketch in Fig. 1(a), and then leads to an asymmetry in the detected pulse, in accordance with our observations. In contrast, when the photoinduced stress field is restricted to the pump skin depth, due to slow (subsonic) diffusion of the initially photoexcited carriers, the emitted strain pulse is a bipolar strain pulse (as detailed at the beginning of this section) and leads to a symmetrically detected pulse, which is also in accordance with the amorphous-state signal.

\section{SUMMARY AND PERSPECTIVES}

Thus, by using a photoacoustic method, we find evidence of a drastic dependence of the spectrum of photoinduced strain on the crystalline state of the phasechange alloy. Analysis of the photoinduced strain profile provides insights into coupling between photoexcited carriers and the lattice. In effect, it remains localized within the photoexcited volume in amorphous $a$-GeTe, indicating that photoexcited carriers release their energy to the lattice within the volume of light absorption. However, in the crystalline $\alpha$-GeTe phase, the photoinduced strain is not local, i.e., not limited to the volume of light absorption. Surprisingly, part of the energy is transported over a distance around 7 times longer than the initial penetration depth of light. Our analysis shows that this effect can be attributed to supersonic diffusion of photoexcited carriers. These results demonstrate that the transport of energy is faster than would be expected if it were solely controlled by heat-transfer processes. This nonthermal transport of energy in GeTe at the picosecond timescale provides important insights into the complex out-of-equilibrium properties of photoexcited crystalline $\mathrm{GeTe}$, which are under debate $[12,16,17]$. The existence of this channel for the transport of energy driven by photoexcited carriers might have important implications for the ultrafast photoassisted amorphization process, the electronic contributions (i.e., nonthermal processes) of which, versus the thermal effect, have not been evaluated to date [16]. Furthermore, PCMs have recently been used in active plasmonic devices [54] and in metamaterials and metalenses [55,56] with, for the latter case, PCM nanostructure dimensions ranging from hundreds of nanometers to submicrometers. Such optical metamaterial efficiency greatly depends on the dielectric function of PCMs. Our findings have a direct consequence on these submicrometer devices, since they show that energy deposited within a typical picosecond timescale after the light-matter interaction is not limited to the optical skin depth $(20-30$ $\mathrm{nm})$ but takes place over hundreds of nanometers, i.e., similar to the submicrometer dimensions of these metamaterials. This might affect the imaginary and real parts of the dielectric constant of such hybrid devices, when driven by light and when interactions with plasmonic antenna take place [56]. Moreover, optical losses are an important issue for PCM-based optical metamaterials. Part of these losses is governed by electron-phonon collision. Showing that photoexcited carriers can diffuse quite efficiently in crystalline $\mathrm{GeTe}$ is an indication of a clear reduction of electron-phonon coupling in crystalline GeTe compared with the amorphous phase. This information can be useful for the development of these optical metamaterials. Finally, considering the picosecond dynamics of this superdiffusive process, our findings could have real implications for the development of sub-THz or THz switching technologies and, in particular, for ultrafast processes in phase-change-material-based technologies.

\section{ACKNOWLEDGMENTS}

This work is partially funded by the project OptoAc of Le Mans Acoustique (Région Pays de la Loire), the 
European Regional Development Fund (FEDER), and the ANR UP-DOWN (Grant No. ANR-18-CE09-0026-04). P.R. thanks Olivier Thomas for fruitful discussions.

[1] B. Gholipour, The promise of phase-change materials, Science 366, 186 (2019).

[2] C. D. Wright, Y. Liu, K. I. Kohary, M. M. Aziz, and R. J. Hicken, Arithmetic and biologically inspired computing using phase change materials, Adv. Mater. 23, 3408 (2011).

[3] P. Hosseini, A. Sebastian, N. Papandreou, C. D. Wright, and $\mathrm{H}$. Bhaskaran, Accumulation-based computing using phase-change memories with FET access device, IEEE Electron Device Lett. 36, 975 (2015).

[4] J. Feldmann, M. Stegmaier, N. Gruhler, C. Ríos, H. Bhaskaran, C. D. Wright, and W. H. P. Pernice, Calculating with light using a chip-scale all-optical abacus, Nat. Commun. 8, 1256 (2017).

[5] N. Gong, T. Idé, S. Kim, I. Boybat, A. Sebastian, V. Narayanan, and T. Ando, Signal and noise extraction from analog memory elements for neuromorphic computing, Nat. Commun. 9, 2102 (2018).

[6] M. Jafari, L. J. Guo, and M. A. Rais Zadeh, Reconfigurable color reflector by selective phase change of GeTe in a multilayer structure, Adv. Opt. Mater. 7, 1801214 (2019).

[7] Z. Cheng, C. Ríos, W. H. P. Pernice, C. D. Wright, and H. Bhaskaran, On-chip photonic synapse, Sci. Adv. 3, e1700160 (2017).

[8] J. Feldmann, N. Youngblood, C. D. Wright, H. Bhaskaran, and W. H. P. Pernice, All-optical spiking neurosynaptic networks with self-learning capabilities, Nature 569, 208 (2019).

[9] M. Le Gallo, A. Sebastian, R. Mathis, M. Manica, H. Giefers, T. Tuma, C. Bekas, A. Curioni, and E. Eleftheriou, Mixed-precision in-memory computing, Nat. Electron. 1, 246 (2018).

[10] C. Ríos, N. Youngblood, Z. Cheng, M. Le Gallo, W. H. P. Pernice, C. D. Wright, A. Sebastian, and H. Bhaskaran, Inmemory computing on a photonic platform, Sci. Adv. 5, 1 (2019).

[11] M. Wuttig and N. Yamada, Phase-change materials for rewriteable data storage, Nat. Mater. 6, 824 (2007).

[12] A. V. Kolobov, P. Fons, A. I. Frenkel, A. L. Ankudinov, J. Tominaga, and T. Uruga, Understanding the phase-change mechanism of rewritable optical media, Nat. Mater. 3, 703 (2004).

[13] M. Wuttig, H. Bhaskaran, and T. Taubner, Phase change materials for non-volatile photonic applications, Nat. Photonics 11, 465 (2017).

[14] S. Raoux, W. Welnic, and D. Ielmini, Phase change materials and their application to nonvolatile memories, Chem. Rev. 110, 240-267 (2010).

[15] C. Ríos, M. Stegmaier, P. Hosseini, D. Wang, T. Scherer, C. D. Wright, H. Bhaskaran, and W. H. P. Pernice, Integrated all-photonic non-volatile multi-level memory, Nat. Photonics 9, 725 (2015).

[16] P. Fons, H. Osawa, A. V. Kolobov, T. Fukaya, M. Suzuki, T. Uruga, N. Kawamura, H. Tanida, and J. Tominaga,
Photoassisted amorphization of the phase-change memory alloy $\mathrm{Ge}_{2} \mathrm{Sb}_{2} \mathrm{Te}_{5}$, Phys. Rev. B 82, 041203(R) (2010).

[17] M. Hada, W. Oba, M. Kuwahara, I. Katayama, T. Saiki, J. Takeda, and K. G. Nakamura, Ultrafast time-resolved electron diffraction revealing the nonthermal dynamics of nearUV photoexcitation-induced amorphization in $\mathrm{Ge}_{2} \mathrm{Sb}_{2} \mathrm{Te}_{5}$, Sci. Rep. 5, 13530 (2015).

[18] M. Hase, P. Fons, A. V. Kolobov, and J. Tominaga, Coherent gigahertz phonons in $\mathrm{Ge}_{2} \mathrm{Sb}_{2} \mathrm{Te}_{5}$ phase-change materials, J. Phys.: Condens. Matter 27, 485402 (2015).

[19] L. Waldecker, T. A. Miller, M. Rudé, R. Bertoni, J. Osmond, V. Pruneri, R. E. Simpson, R. Ernstorfer, and S. Wall, Time-domain separation of optical properties from structural transitions in resonantly bonded materials, Nat. Mater. 14, 991 (2015).

[20] P. Zalden, F. Quirin, M. Schumacher, J. Siegel, S. Wei, A. Koc, M. Nicoul, M. Trigo, P. Andreasson, H. Enquist et al., Femtosecond X-ray diffraction reveals a liquid-liquid phase transition in phase-change materials, Science 14, 1062 (2019).

[21] P. Fons, P. Rodenbach, K. V. Mitrofanov, A. V. Kolobov, J. Tominaga, R. Shayduk, A. Giussani, R. Calarco, M. Hanke, H. Riechert et al., Picosecond strain dynamics in $\mathrm{Ge}_{2} \mathrm{Sb}_{2} \mathrm{Te}_{5}$ monitored by time-resolved X-ray diffraction, Phys. Rev. B 90, 094305 (2014).

[22] C. Thomsen, H. T. Grahn, H. J. Maris, and J. Tauc, Surface generation and detection of phonons by picosecond light pulses, Phys. Rev. B 34, 4129 (1986).

[23] V. Gusev and A. Karabutov, Laser Optoacoustics (AIP, New York, 1993).

[24] P. Ruello and V. E. Gusev, Physical mechanisms of coherent acoustic phonons generation by ultrafast laser action, Ultrasonics 56, 21 (2015).

[25] O. B. Wright, B. Perrin, O. Matsuda, and V. E. Gusev, Ultrafast carrier diffusion in gallium arsenide probed with picosecond acoustic pulses, Phys. Rev. B 64, 081202 (2001).

[26] E. S. K. Young, A. V. Akimov, R. P. Campion, A. J. Kent, and V. Gusev, Picosecond strain pulses generated by a supersonically expanding electron-hole plasma in GaAs, Phys. Rev. B 86, 155207 (2013).

[27] N. Chigarev, D. Yu Paraschuk, X. Y. Pan, and V. Gusev, Coherent acoustic emission in the supersonic expansion of the photogenerated electron-hole plasma, Phys. Rev. B 61, 15837 (2000).

[28] G. Tas and H. J. Maris, Electron diffusion in metals studied by picosecond ultrasonics, Phys. Rev. B 49, 15046 (1994).

[29] M. Lejman, V. Shalagatskyi, O. Kovalenko, T. Pezeril, V. V. Temnov, and P. Ruello, Ultrafast optical detection of coherent acoustic phonons emission driven by superdiffusive hot electrons, J. Opt. Soc. Am. B 31, 282 (2014).

[30] P. Ruello, A. Ayouch, G. Vaudel, T. Pezeril, N. Delorme, S. Sato, K. Kimura, and V. E. Gusev, Ultrafast acoustoplasmonics in gold nanoparticle superlattices, Phys. Rev. B 92, 174304 (2015).

[31] M. Först, T. Dekorsy, C. Trappe, M. Laurenzis, H. Kurz, and $\mathrm{B}$. Béchevet, Phase change in $\mathrm{Ge}_{2} \mathrm{Sb}_{2} \mathrm{Te}_{5}$ films investigated by coherent phonon spectroscopy, Appl. Phys. Lett. 77, 1964 (2000).

[32] J. Hernandez-Rueda, A. Savoia, W. Gawelda, J. Solis, B. Mansart, D. Boschetto, J. Siegel, et al., Coherent optical 
phonons in different phases of $\mathrm{Ge}_{2} \mathrm{Sb}_{2} \mathrm{Te}_{5}$ upon strong laser excitation, Appl. Phys. Lett. 98, 251906 (2011).

[33] M. J. Shu, I. Chatzakis, Y. Kuo, P. Zalden, and A. M. Lindenberg, Ultrafast sub-threshold photo-induced response in crystalline and amorphous GeSbTe thin films, Appl. Phys. Lett. 102, 201903 (2013).

[34] J. Siegel, W. Gawelda, D. Puerto, C. Dorronsoro, J. Solis, C. N. Afonso, J. C. G. de Sande, R. Bez, A. Pirovano, and $\mathrm{C}$. Wiemer, Amorphization dynamics of $\mathrm{Ge}_{2} \mathrm{Sb}_{2} \mathrm{Te}_{5}$ films upon nano- and femtosecond laser pulse irradiation, J. Appl. Phys. 103, 023516 (2008).

[35] See the Supplemental Material at http://link.aps.org/sup plemental/10.1103/PhysRevApplied.16.014055 for the determination of the subthreshold condition to realize picosecond acoustics experiments in a perturbative regime (Note S1 [18]), ultrafast carrier dynamics (Note S2 [40, 41]), determination of the wavelength dependence of the photoelastic coefficients (Note S3 $[13,46]$ ), determination of the sound velocity (Note S4), and estimation of the nonlinear acoustic effect (Note S5 [23,46,57-59]).

[36] K. S. Andrikopoulos, S. N. Yannopoulos, G. A. Voyiatzis, A. V. Kolobov, M. Ribes, and J. Tominaga, Raman scattering study of the $a$-GeTe structure and possible mechanism for the amorphous to crystal transition, J. Phys.: Condens. Matter 18, 965 (2006).

[37] A. V. Kolobov, P. Fons, J. Tominaga, A. I. Frenkel, A. L. Ankudinov, S. N. Yannopoulous, K. S. Andrikopoulos, and T. Uruga, Why phase-change media are fast and stable: A new approach to an old problem, Jpn. J. Appl. Phys. 44, 3345 (2005).

[38] P. Ruello, S. Zhang, P. Laffez, B. Perrin, and V. Gusev, Laser-induced coherent acoustic phonon mechanisms in metal-insulator compounds $\mathrm{NdNiO}_{3}$ : Thermal and nonthermal processes, Phys. Rev. B 79, 094303 (2009).

[39] M. Schubert, H. Schaefer, J. Mayer, A. Laptev, M. Hettich, M. Merklein, C. He, C. Rummel, O. Ristow, M. Großmann et al., Collective Modes and Structural Modulation in Ni$\mathrm{Mn}-\mathrm{Ga}(\mathrm{Co})$ Martensite Thin Films Probed by Femtosecond Spectroscopy and Scanning Tunneling Microscopy, Phys. Rev. Lett. 115, 076402 (2015)

[40] P. Ruello, S. Zhang, P. Laffez, B. Perrin, and V. Gusev, Ultrafast electronic dynamics in the metal-insulator transition compound $\mathrm{NdNiO}_{3}$, Phys. Rev. B 76, 165107 (2007).

[41] J. Demsar, K. Biljakovic, and D. Mihailovic, Single Particle and Collective Excitations in the One-Dimensional Charge Density Wave Solid $\mathrm{K}_{0.3} \mathrm{MoO}_{3}$ Probed in Real Time by Femtosecond Spectroscopy, Phys. Rev. Lett. 83, 800 (1999).

[42] D. Dangic, A. R. Murphy, E. D. Murray, S. Fahy, and I. Savic, Coupling between acoustic and soft transverse optical phonons leads to negative thermal expansion of $\mathrm{GeTe}$ near the ferroelectric phase transition, Phys. Rev. B 97, 224106 (2018).

[43] R. Shaltaf, E. Durgun, J.-Y. Raty, Ph. Ghosez, and X. Gonze, Dynamical, dielectric, and elastic properties of GeTe investigated with first-principles density functional theory, Phys. Rev. B 78, 205203 (2008).

[44] A. Onodera, I. Sakamoto, Y. Fujii, N. Môri, and S. Sugai, Structural and electrical properties of GeSe and GeTe at high pressure, Phys. Rev. B 56, 7935 (1997).
[45] P. B. Pereira, I. Sergueev, S. Gorsse, J. Dadda, E. Müller, and R. 1. P. Hermann, Lattice dynamics and structure of GeTe, SnTe and PbTe, Phys. Status Solidi B 250, 1300 (2013).

[46] J.-W. Park, S. H. Baek, T. D. Kang, H. Lee, Y.-S. Kang, T.-Y. Lee, D.-S. Suh, K. J. Kim, C. K. Kim, Y. H. Khang et al., Optical properties of $\left(\mathrm{GeTe}, \mathrm{Sb}_{2} \mathrm{Te}_{3}\right)$ pseudobinary thin films studied with spectroscopic ellipsometry, Appl. Phys. Lett. 93, 021914 (2008).

[47] X. Shen, Z. Lu, Y. P. Timalsina, T.-M. Lu, M. Washington, and M. Yamaguchi, Coherent phonon transport measurement and controlled acoustic excitations using tunable acoustic phonon source in $\mathrm{GHz}$-sub $\mathrm{THz}$ range with variable bandwidth, Sci. Rep. 8, 7054 (2018).

[48] R. Shaltaf, X. Gonze, M. Cardona, R. K. Kremer, and G. Siegle, Lattice dynamics and specific heat of -GeTe: Theoretical and experimental study, Phys. Rev. B 79, 075204 (2009).

[49] D. Campi, L. Paulatto, G. Fugallo, F. Mauri, and M. Bernasconi, First-principles calculation of lattice thermal conductivity in crystalline phase change materials: GeTe, $\mathrm{Sb}_{2} \mathrm{Te}_{3}$, and $\mathrm{Ge}_{2} \mathrm{Sb}_{2} \mathrm{Te}_{5}$, Phys. Rev. B 95, 024311 (2017).

[50] S. A. Akhmanov and V. E. Gusev, Laser excitation of ultrashort acoustic pulses: New possibilities in solid-state spectroscopy, diagnostics of fast processes, and nonlinear acoustics, Sov. Phys. Usp. 35, 153 (1992).

[51] S. Adachi, GaAs and Related Materials, Bulk Semiconductors and Superlattice Properties (World Scientific, Singapore, 1994).

[52] Z. Liu, J. Sun, J. Mao, H. Zhu, W. Ren, J. Zhou, Z. Wang, D. J. Singh, J. Sui, C.-W. Chu et al., Phase-transition temperature suppression to achieve cubic GeTe and high thermoelectric performance by $\mathrm{Bi}$ and $\mathrm{Mn}$ codoping, Proc. Natl. Acad. Sci. 115, 5332 (2018).

[53] C. Longeaud, J. Luckas, D. Krebs, R. Carius, J. Klomfass, and M. Wuttig, On the density of states of germanium telluride, J. Appl. Phys. 112, 113714 (2012).

[54] N. Farmakidis, N. Youngblood, X. Li, J. Tan, J. L. Swett, Z. Cheng, C. D. Wright, W. H. P. Pernice, and H. Bhaskaran, Plasmonic nanogap enhanced phase-change devices with dual electrical-optical functionality, Sci. Adv. 5, 2687 (2019).

[55] M. Y. Shalaginov, S. An, Y. Zhang, F. Yang, P. Su, V. Liberman, J. B. Chou, C. M. Roberts, M. Kang, C. Rios et al., Reconfigurable all-dielectric metalens with diffraction-limited performance, Nat. Commun. 12, 1225 (2021).

[56] S. Abdollahramezani, O. Hemmatyar, H. Taghinejad, A. Krasnok, Y. Kiarashinejad, M. Zandehshahvar, A. Alù, and A. Adibi, Tunable nanophotonics enabled by chalcogenide phase-change materials, Nanophotonics 9, 1189 (2020).

[57] P. J. S. van Capel, E. Péronne, and J. I. Dijkhuis, Nonlinear ultrafast acoustics at the nanoscale, Ultrasonics 56, 36 (2015).

[58] S. Rajagopalan and D. N. Joharapurkar, Temperature dependence of the nonlinearity constant and ultrasonic attenuation in pure silicon and germanium, J. Appl. Phys. 54, 3166 (1983).

[59] H. Wiedemeier and P. A. Siemers, The thermal expansion of GeS and GeTe, Z. Anorg. Allg. Chem. 431, 299 (1977). 